\title{
Imprint of Distortions in the Oort Cloud on the CMB Anisotropies
}

\author{
Daniel Babich ${ }^{a}$ Abraham Loeb ${ }^{b}$ \\ a California Institute of Technology, Theoretical Astrophysics, MC 130-33 \\ Pasadena, CA 91125, USA \\ b Astronomy Department, Harvard University, 60 Garden Street, Cambridge, MA \\ 02138, USA
}

\begin{abstract}
We study the effect of a close encounter of a passing star on the shape of the inner Oort Cloud, using the impulse approximation. The deviation of the perturbed Oort Cloud from sphericity adds angular fluctuations to the brightness of the Cosmic Microwave Background (CMB) due to thermal emission by the comets. An encounter with a solar-mass star at an impact parameter of $2100 \mathrm{AU}$, as expected based on the abundance and velocity dispersion of stars in the solar neighborhood, leads to a quadrupole moment $C_{2}=4.5(3.5) \times 10^{-15}, 6.7(1.1) \times 10^{-12}, 1.1(0.11) \times 10^{-9}$ at $\nu=30,353,545 \mathrm{GHz}$, respectively in intensity and (temperature) fluctuations. We also quantify the quadrupole spectral distortions produced by the Scattered Disc, which will exist irregardless of any perturbation and the subsequent shape of the Oort Cloud. For comparison, the temperature fluctuation quadrupole moment predicted by the current cosmological model is $C_{2}=1.76 \times 10^{-10}$, which corresponds to fluctuation in the CMB intensity of $C_{2}=2.9 \times 10^{-10}, 6.8 \times 10^{-9}, 1.6 \times 10^{-8}$ at $\nu=30,353,545 \mathrm{GHz}$. Finally, we discuss how a measurement of the anisotropic spectral distortions could be used to constrain the trajectory of the closest stellar fly-by.
\end{abstract}

Key words: cosmic microwave background, Oort Cloud 96.50.Hp, 98.70.Vc

\section{Introduction}

At the outer edge of the Solar System exists a swarm of small icy rocks known as the Oort Cloud (Oort, 1950; Dones et al., 2004). Comets with long orbital

Email address: babich@tapir.caltech.edu (Daniel Babich). 
periods, $P \geq 200 \mathrm{yr}$, are ejected from this cloud into the inner Solar System by the perturbations from passing stars, giant molecular clouds or tides from the Galactic disc. The perturbed comets are moved onto nearly-parabolic orbits which approach the Sun, develop comae and may be detected. Gravitational perturbations by the planets can strongly alter the comets' orbits as they enter the inner Solar System, and so typically they either get ejected from the Solar System or left strongly bound to the Sun. It is very unlikely that a comet will return again on a nearly parabolic orbit. The observed continual flux of long period comets on nearly parabolic orbits originally led Oort (1950) to propose that all long period comets were entering the inner Solar System for the first time and therefore a resevoir of comets must exists in the outermost region of the Solar System.

There is considerable interest in studying the Oort Cloud since it is a remnant of the formation epoch of the Solar System. This is true for both the individual objects, whose chemical composition may reveal information about the composition and thermal state of the outer regions of the proto-planetary disc (but see Mumma et al. (1993) for a comprehensive review of processes that may cause significant evolution in these properties over the past $4 \mathrm{Gyr}$ ) as well as the dynamical structure of the Oort Cloud as a whole. The cloud structure, which is largely determined by its formation scenario, may retain information about the masses of the giant planets, the rate at which passing stars perturbed the comets' orbits and the surface density of any gas that was potentially still within the Solar System. This information could provide important clues about the formation processes of the outer planets.

The Oort Cloud is cruedly divided into an inner and an outer region. This division stems from the fact that the probability for ejection into the inner Solar System by external perturbations is a strong function of semi-major axis (Heisler \& Tremaine, 1986; Dones et al., 2004). All of the observed longperiod comets are believed to have originated from the outer Oort Cloud. The perturbations that eject comets into the inner Solar System also isotropize the comets' orbits and eliminates any information about the formation epoch in the structure of the outer Oort Cloud. Therefore the reduced sensitivity of the comets in the inner Oort Cloud to these external perturbers is both good and bad. On the one hand it leads us to suspect that the structure of the inner Oort Cloud should retain an imprint of its formation as well as any rare, strong gravitational scattering event that might have occured over the past 4.5 billion years. On the other hand, it makes studying the inner Oort Cloud difficult. It is impossible to directly observe the comets in the inner Oort Cloud through their reflected sunlight because they are too far. Extremely large objects like Sedna can be optically detected when they are close to perihelion (Brown et al., 2004), however it will be difficult to detect the potentially large number of small objects $(R \leq 1 \mathrm{~km})$ in this region. One novel technique involves using the microwave emission from these bodies to 
constrain, or possibly detect, the bulk properties of objects in this region (Babich et al., 2007).

At a distance $D$ from the sun an Oort Cloud object will be heated by the Sun to an equilibrium temperature,

$$
\begin{aligned}
T_{\text {Oort }} & =\left(\frac{(1-A) L_{\odot}}{16 \epsilon \sigma \pi D^{2}}\right)^{1 / 4} \\
& \simeq 8.5 \mathrm{~K} \sqrt{\frac{1000 \mathrm{AU}}{D}},
\end{aligned}
$$

here we adopted an albedo of $A=4 \%$ (Jewitt \& Luu, 1998) and an emissivity of $\epsilon \simeq 1.0$. We adopt a model such that the emissivity of an Oort Cloud object is approximately unity when its size is larger than the wavelength of the relevant radiation and zero when the wavelength is larger. Also, $\sigma$ is the Stefan-Boltzmann constant and $L_{\odot}=3.83 \times 10^{33} \mathrm{ergs} \mathrm{s}^{-1}$ is the Solar luminosity. The equilibrium temperature is higher than the Cosmic Microwave Background $(\mathrm{CMB})$ temperature of $T_{\mathrm{CMB}}=2.725 \mathrm{~K}$ (Mather et al., 1999). When the comets are farther away from the Sun a variety of physical processes help maintain temperatures greater than 5-6 K (Mumma et al., 1993). The Oort Cloud objects extinguish some fraction of the CMB radiation and emit blackbody radiation at a higher temperature (Babich et al., 2007). The observed intensity of the $\mathrm{CMB}$ radiation is then

$$
I_{\nu}(\hat{\boldsymbol{n}})=[1-\tau(\hat{\boldsymbol{n}})] B_{\nu}\left[T_{\mathrm{CMB}}(\hat{\boldsymbol{n}})\right]+\tau(\hat{\boldsymbol{n}}) B_{\nu}\left[T_{\mathrm{Oort}}(\hat{\boldsymbol{n}})\right]
$$

where $\tau$ is the optical depth and $T_{\text {Oort }}$ is temperature of the Oort Cloud objects along the direction $\hat{\boldsymbol{n}}$. Babich et al. (2007) constrained the mean, or monopole, contribution to the CMB spectrum using the Cosmic Background Explorer Far Infrared Absolute Spectrophotometer (COBE FIRAS) data (Fixsen et al., 1997; Mather et al., 1999) and placed upper limits on the total mass in the inner Oort Cloud for a variety of models of the size distribution of Oort Cloud objects. In this paper we will focus on effects that can make the Oort Cloud non-spherical and subsequently produce anisotropic spectral distortions in the CMB.

We should emphasize that the induced spectral distortions considered in this paper are not the chemical potential $(\mu)$ or Compton-y spectral distortion that are commonly discussed in the literature. The signal produced in the outer Solar System is that of a sum of weighted blackbodies at different temperatures, which in general does not have a blackbody frequency spectrum. The exception is the Rayleigh-Jean's portion of the spectrum, where the weighted sum of blackbodies does produce a frequency spectrum described by a RayleighJean's distribution at a weighted temperature. This implies that low frequency data will not be very useful in allowing us to detect a signal originating from 
the outer Solar System. See Babich et al. (2007) for a discussion of techniques to distinguish the spectral distortions produced in the outer Solar System from those caused by the CMB temperature anisotropies.

The Oort Cloud is believed to be nearly spherical based on the statistics of the inclination angles of new long period comets (Marsden \& Sekanina, 1971). The observed apsides of these comets are not uniformly distributed across the sky, however the observed clustering trends are believed to be due to the structure of the Galactic potential (Delsemme, 1987; Dones et al., 2004). The Oort Cloud has been populated by objects that formed in the Sun's protoplanetary disc and were scattered multiple times by the four giant planets into increasingly higher eccentricity and higher energy orbits. This process continued until some external perturbation lifted the comets' perihelia well beyond Neptune's orbit, and then the comets became decoupled from the inner Solar System. The radial distribution of the Oort Cloud comets, which is set by how far the gas giant planets can scatter the comets before their perihelia is lifted safely outside the inner Solar System, strongly depends on the external environment of the young Solar System. The evolutionary processes that lifted the perihelia of comets beyond the planetary region of the Solar System must have also made shperical the distribution of comets which where initially in the Ecliptic Plane. Simulations of this formation process have found an inner edge of the Oort Cloud, defined as the semi-major axis at which the comets are isotropically distributed, near $a=3000 \mathrm{AU}$ (Duncan et al., 1987) and $a=5000 \mathrm{AU}$ (Dones et al., 2007). It should be emphasized that objects with smaller semi-major axes exist, but may be more confined towards the Ecliptic Plane. This is the Scattered Disc which smoothly extends from the flattened Kuiper Belt to the spherical Oort Cloud. We will quantify the effect of the Scattered Disc by calculating the quadrupole moments of its thermal emission. This will exist irregardless of any externally induced asphericity in the Oort Cloud.

An increasing body of evidence now suggests that the Solar System did not form in isolation, but in a stellar cluster which subsequently evaporated (Adams \& Laughlin, 2001; Hester et al., 2004). This is not surprising since most low mass stars are observed to form in clusters that subsequently evaporate when the interstellar gas, which is required to gravitational bind the cluster, is expelled from the cluster by either stellar winds or supernova feedback (Lada \& Lada, 2003). Beyond this general trend, anomalous abundance ratios are observed in the Solar System that would indicate that a Type II supernova occur somewhere in the vicinity of the Solar System during its infancy (Looney et al., 2006). With a higher number density of stars in the cluster the rate of external perturbations would have been higher and the spherical inner edge of the Oort Cloud would have extended to smaller semi-major axes.

In this paper we will consider the dynamical effect that a star passing within 
$\lesssim 1 \mathrm{pc}$ of the sun would have had on the Oort Cloud. And we calculate the signatures that would be produced in the CMB frequency spectrum. The outline of this paper is as follows. In $\$ 2$ we describe how a non-sherical distribution of Oort Cloud objects may affect the measured brightness distribution of the $\mathrm{CMB}$ on the sky. In $\$ 2.1$ we calculate the variance of the Oort Cloud signal due to Poisson fluctuations, whereas in $\$ 2.2$ we describe the initial distribution of Oort Cloud objects. In $\$ 2.3$ we outline of method of calculation the perturbations in the orbital elements of the Oort Cloud objects, and in \$2.4 we calculate the distribution of impact parameters of stellar perturbers. In $\$ 3$ we derive an formula based on the collisionless Boltzmann equation that allows us to analytically estimate the resulting quadrupole distortion from a perturbation. Our results are discussed in \$4 In \$5 we develop a Bayesian estimator that can be used to constrain the parameters of a stellar encounter from the observed CMB data. Finally $\$ 6$ summarizes our main conclusions, Appendix $\mathrm{A}$ derives relationships between various measures the quadrupole

moment and Appendix B describes the model of instrumental noise for the Planck satellite.

\section{Calculation}

We start by calculating how the non-sphericity of the Oort Cloud affects the measured angular distribution of the CMB intensity. The fluctuation in the CMB frequency spectrum along a given direction

$$
\delta I_{\nu}(\hat{\boldsymbol{n}}) \equiv I_{\nu}(\hat{\boldsymbol{n}})-B_{\nu}\left(\bar{T}_{\mathrm{CMB}}\right),
$$

can be expressed as

$$
\begin{aligned}
\delta I_{\nu}(\hat{\boldsymbol{n}})= & \Delta T(\hat{\boldsymbol{n}}) \frac{\partial B_{\nu}(T)}{\partial T} \\
& +f_{A} \int d x_{i} p\left(x_{i}\right) \frac{1}{D\left(x_{i}\right)^{2}}\left[B_{\nu}\left(T_{\mathrm{Oort}}\left(x_{i}\right)\right)-B_{\nu}\left(\bar{T}_{\mathrm{CMB}}\right)\right]
\end{aligned}
$$

where $p\left(x_{i}\right)$ is the probability distribution of orbital elements describing the Keplerian orbits of the Oort Cloud objects around the Sun. The first term on the right is due to the extragalactic CMB temperature anisotropies. The CMB signal that comes from the surface of last scattering $(z=1000)$ has a blackbody frequency distribution that is parameterized by slightly different temperatures in different directions (Dodelson, 2003). Once we have subtracted off the frequency spectrum corresponding to the average CMB temperature, Eq. (44), the CMB temperature anisotropies induce some spectral distortion since we have removed a blackbody at an incorrect temperature for that particular pixel. The second term is caused by the Oort Cloud objects in the outer Solar 
System as described in $\S \mathbb{1}$, where $D$ is the distance of the Oort Cloud object from the Sun. The absolute covering fraction is

$$
f_{A}=\int d M \frac{R^{2}}{4} \frac{d n}{d M}(M)
$$

where $R$ is the radius of the Oort Cloud object. The absolute covering fraction is related to the optical depth as $\tau=f_{A} / D^{2}$. Since the individual objects are optically thick, but sparsely fill the telescope beam function with which the CMB sky has been observed, the optical depth is simply related to the covering fraction of Oort Cloud objects. We add the term 'absolute' because $f_{A}$ is the covering fraction if all Oort Cloud objects were placed at a standard distance. We have implicitly assumed that the physical size and the orbital elements of the Oort Cloud objects are uncorrelated. This assumption allows us to decouple the probability distribution of the orbital elements used in Eq. (5) from the size distribution, defined in Eq. (7) and used to calculate the absolute covering fraction. This is a reasonable assumption since the comets behave as test particles with respect to Sun and the perturbing stan 1 . For the internal density of the Oort Cloud objects we adopt a value of $\rho=1 \mathrm{~g} \mathrm{~cm}^{-3}$.

In order to relate the mean optical depth to a total mass of the inner Oort Cloud, the differential mass function must be specified. We model it as a broken power-law 2

$$
\begin{array}{ll}
\frac{d n}{d M}(M)=A M^{-\alpha}, & M_{\min }<M<M_{\mathrm{br}}, \\
\frac{d n}{d M}(M)=A M_{\mathrm{br}}^{-\alpha+\beta} M^{-\beta}, & M_{\mathrm{br}}<M<M_{\max } .
\end{array}
$$

The mass distribution yields the total mass through the integral

$$
M_{\mathrm{tot}}=\int d M M \frac{d n}{d M}(M) .
$$

The empirical values of the above power-law indices are not known. For the Kuiper Belt, the slope of the high mass distribution has been restricted to the

$\overline{1}$ Note that it is not obvious that the initial distribution of orbital elements should be independent of the object's mass because non-gravitational forces, such as gas drag (Weidenschilling, 1977; Rafikov, 2004) and Pointying-Robertson drag (Burns et al., 1979), may have been important when the gas giant planets were scattering the comets into highly eccentric orbits. Also, collisional effects, which are more important for small objects, may alter the formation of the Oort Cloud (Stern \& Weissman, 2001; Charnoz \& Morbidelli, 2003, 2006). These are issues that go beyond the scope of this paper.

2 Typically the differential mass function is expressed in terms of comet radius, not mass. The power-law index with respect to radius $\alpha_{\text {radius }}$ is related to the values used in this paper as $\alpha_{\text {mass }}=\left(\alpha_{\text {radius }}+2\right) / 3$. 
range $\beta=2 \pm 0.2$ (Bernstein et al., 2004). There are no strong observational constraints on the slope of the low mass distribution, $\alpha$; however, it can be calculated through the assumption of collisional equilibrium (Pan \& Sari, 2005). The exact value of the slope then depends on the assumed material strength of the Kuiper Belt objects. Similiar constraints on the inner Oort Cloud mass distribution does not exist. Since collisions have not been numerous enough to affect the slope of the high mass distribution, it is reasonable to adopt the observed value of the slope of planetesimals in the proto-planetary disc and use it for the distribution of inner Oort Cloud objects. We will adopt $\beta=2.16$ for the calculations in this paper. The collisional grinding of the Kuiper Belt objects, which justifies the above assumption of collisional equilibrium, is believed to have occurred amongst the objects at their current location. The collision timescales in the Oort Cloud are much longer, so a state of collisional equilibrium cannot have been reach if the objects were always at their present locations. Fortunately collisions are believed to have been frequent during the formation phase of the Oort Cloud while the objects were still in the planetary region of the Solar System (Stern \& Weissman, 2001; Charnoz \& Morbidelli, 2003, 2006). Again we will adopt the Kuiper Belt value of $\alpha=1.83$. Our choice of this value is conservative, since the primordial slope was likely steeper, providing even better constraints than we will derive.

We assume $M_{\max } \equiv M_{\text {Sedna }}=6 \times 10^{25} \mathrm{~g}$ (Brown, 2007), as the upper mass limit. Our results are insensitive to changes in the upper mass limit for $\beta>2$. We will take the minimum mass to correspond to objects of radius $1 \mathrm{~mm}$. This quantity depends on the detailed formation history of the Oort Cloud and is, therefore difficult to calculate from first principles. The minimum object mass is partially degenerate with the total mass of the outer Solar System which is also unknown, so for the purposes of this paper we will fix the minimum radius at $1 \mathrm{~mm}$. However, we note that the efficiency of blackbody emission is suppressed when the radiation wavelength is larger than the size of object emitting the radiation due to Kirchoff's Law (Greenberg, 1978; Backman \& Paresce, 1993; Landau \& Lifshitz, 1999). It should be emphasized that we only need our adopted model of the size distribution to convert a total mass of the Oort Cloud into an optical depth; if we simply choose a value for the optical depth then the rest of this paper would be independent of our assumed size distribution.

For the location of the break in our mass model, we assume $M_{\mathrm{br}}=4 \times 10^{15} \mathrm{~g}$ (corresponding to $R_{\mathrm{br}}=1 \mathrm{~km}$ ). This value is established by requiring that objects of this size were likely to have undergone a collision during the time period of Oort Cloud's formation. The formation of the Oort Cloud most likely was a continuous process so it is difficult to precisely define its formation time. In simulations this time period can roughly be estimated as $\Delta t_{\text {Oort }}=200 \mathrm{Myr}$ based on a visual inspection (see Fig. 8 of Dones et al. (2004)). The collision 
rate of objects of size $R$ in a protoplanetary disc is (Goldreich et al., 2004)

$$
\Gamma=\frac{\sigma \Omega}{\rho R}
$$

where $\sigma$ is the surface mass density of planetesimals, $\Omega$ is the Keplerian velocity and $\rho$ is the planetesimal density. We adopt minimum mass solar nebula value for the surface density of condensates $\sigma=(20 \mathrm{AU} / a)^{3 / 2} \mathrm{~g} \mathrm{~cm}^{-2}$ (Goldreich et al., 2004). Now the break radius can be calculated as

$$
\begin{aligned}
R_{\mathrm{br}} & =\frac{\sigma \Omega \Delta t_{\text {Oort }}}{\rho} \\
& =\frac{1 \mathrm{~km}}{(a / 20 \mathrm{AU})^{3}} .
\end{aligned}
$$

A full simulation would be required to precisely determine this quantity as well as its radial dependence within the Oort Cloud. This is roughly consistent with the HST results of Bernstein et al. (2004) for the Kuiper Belt. Our results are insensitive to the precise choice of these values since they are dominated by the smallest objects which have the largest surface area to volume ratio.

The total mass in the Oort Cloud is calculated by requiring there to be $10 M_{\oplus}$ in objects $R \geq 1 \mathrm{~km}$ in the outer Oort Cloud (Weissman, 1990). This figure is related to the observed number of new long period comets and implies there is approximately $10^{13}$ comets present in the outer Oort Cloud. We use our adopted models to relate this number of comets to the total mass in the inner Oort Cloud. For simplicity we assume equal amounts of mass in the inner and outer Oort Clouds. Simulations of the formation of the Oort Cloud have implied that the inner Oort Cloud is more massive (Duncan et al., 1987), while others have implied that the outer Oort Cloud is more massive (Dones et al., 2007). This uncertainity, as well as the uncertainity in other aspects of our model, should be remembered when interpreting our results. In Table 1 we present slightly different models and calculate the total number of objects, the total mass and the effective optical depths of these models. We quote the absolute covering fraction $\left(f_{A}\right)$ divided by the mean squared distance

$$
\frac{1}{\left\langle a^{2}\right\rangle}=\int d a P(a) \frac{1}{a^{2}}
$$

The distribution of semi-major axes and the limits of integration are described in $\oint 2.2$. For definitiveness we adopt $P(a) \propto a^{-3} ; a_{\max }=10^{4} \mathrm{AU}$ and $a_{\min }=$ $10^{3} \mathrm{AU}$ for our calculations in Table 1. These parameter choices lead to the value

$$
\frac{1}{\left\langle a^{2}\right\rangle}=5.05 \times 10^{-7} \mathrm{AU}^{-2}
$$

Next we calculate the microwave anisotropies induced by the thermal emission of the inner Oort Cloud. We consider the real-space quadrupole moments 


\begin{tabular}{cccccc}
\hline \hline$R_{\min }(\mathrm{cm})$ & $R_{\mathrm{br}}(\mathrm{km})$ & $\alpha$ & $N_{\text {Oort }}$ & $M_{\text {total }}\left(M_{\oplus}\right)$ & $\tau=f_{A} /\left\langle a^{2}\right\rangle$ \\
\hline \hline 0.1 & 10 & 1.83 & $6.2 \times 10^{28}$ & 1184 & $1.8 \times 10^{-6}$ \\
0.1 & 1 & 1.83 & $1.0 \times 10^{29}$ & 590 & $2.9 \times 10^{-6}$ \\
0.1 & 10 & 2.0 & $7.1 \times 10^{31}$ & 2565 & $1.2 \times 10^{-3}$ \\
0.01 & 10 & 1.83 & $8.1 \times 10^{31}$ & 1194 & $2.1 \times 10^{-5}$ \\
\hline \hline
\end{tabular}

Table $\overline{\overline{1}}$

Total number $\left(N_{\text {Oort }}\right)$, total mass $\left(M_{\text {total }}\right)$ and absolute covering fraction $\left(f_{A}\right)$ of Oort Cloud objects for the adopted model in this paper (the first row) and slight changes to the model. Also shown is the optical depth $(\tau)$ for each model. We calculate these quantities by requiring that $50 M_{\oplus}$ of comets $(R \geq 1 \mathrm{~km})$ exist.

instead of the more conventional spherical harmonic moments, because it is easier to interpret the results in real space. These moments are defined as

$$
Q_{i j}(\nu)=\int d^{2} \hat{\boldsymbol{n}}\left(\hat{\boldsymbol{n}}_{i} \hat{\boldsymbol{n}}_{j}-\frac{1}{3} \delta_{i j}\right) \frac{\delta I_{\nu}(\hat{\boldsymbol{n}})}{I_{\mathrm{CMB}}}
$$

where $I_{\mathrm{CMB}}=B_{\nu}\left(T_{\mathrm{CMB}}\right)$ is the homogeneous intensity of the $\mathrm{CMB} 3$. We also quote our results as temperature fluctuations, which are more commonly used, in 86 .

There are five independent quadrupole moments because the quadrupole tensor is symmetric and traceless. The Oort Cloud quadrupole moments are also frequency dependent. The standard CMB moments are frequency independent 4 because the usual sources of the cosmological temperature anisotropy, gravitational redshift and Thomson scattering, do not depend on the frequency of the scattered radiation (Dodelson, 2003). Of course, we can relate the real space quadrupole moments to the spherical harmonic moments. These relationships and the conversion between different measures of the quadrupole are discussed in Appendix A.

3 We should note that if the Oort Cloud was intrinsically spherical in a heliocentric coordinate system and the directional vectors used to measure the quadrupole moments, $\hat{\boldsymbol{n}}$ in Eq. (14), were defined in a geocentric coordinate system, then a small quadrupole moment would be observed. This quadrupole moments is roughly $Q \sim\left(r_{\oplus} / r_{\text {Oort }}\right)^{2} \delta I \sim 10^{-6} \delta I$ and therefore quite small.

4 This statement is only true when the primordial CMB moments are expressed in terms on temperature, not intensity, flucuations. The Oort Cloud moments expressed as either temperature or intensity fluctuations are frequency dependent. 


\subsection{Poisson Fluctuations}

The flux emitted by a single unresolved Oort Cloud object scales as

$$
f_{\nu} \propto \frac{1}{D^{2}} B_{\nu}\left[T_{\text {Oort }}(D)\right]
$$

which in the Rayleigh-Jean's portion of the spectrum scales as $f_{\nu} \propto D^{-5 / 2}$. At higher frequencies in the Wien portion of the spectrum the scaling has a steeper dependence on distance $D$. Consequently, even when we calculate the time average of the quadrupole moments

$$
\begin{aligned}
\left\langle f_{\nu}(\hat{\boldsymbol{n}})\right\rangle_{T} & =\frac{1}{P} \int d t f_{\nu}(\hat{\boldsymbol{n}}), \\
& \propto \int d f D^{2} f_{\nu}(\hat{\boldsymbol{n}}),
\end{aligned}
$$

the integrand still scales as an inverse power of $D$. In the above equation we have used Kepler's second law 5 to change integration variables from time $(t)$ to true anomaly $(f)$. In Eq. (16) $P$ is the orbital period of the Oort Cloud object. Therefore, an individual Oort Cloud object will produce the largest amount of signal per unit time when it is closest to perihelion and equivalently the comets that happen to be nearest to their perihelia will contribute the greatest to the overall signal per unit surface area. Since the comet's velocity is largest close to perihelion there will be fluctuations in the direction and amplitude of the quadrupole. These fluctuations will become larger at high frequencies where the signal's scaling with inverse distance is even stronger. The signal in a given direction is the average signal of all Oort Cloud objects weighted by their apparent angular size. If the relatively few nearby objects that appear large, have a higher temperature and move quickly, dominate the signal over the more numerous farther away objects, then the Poisson fluctuations will be larger.

We adopted a continuum description of the Oort Cloud object masses and orbital elements, which implicitly assumed an infinite number of objects, when calculating the induced CMB spectral distortions in $\$ 2$, In reality there are only a finite number of objects along any given line-of-sight and the signal will be dominated by the smaller number of objects that are closest to perihelion, so Poisson fluctuations will produce statistical fluctuations. These fluctuations will be estimated in this section.

The surface brightness in a given direction, which is now a random variable, can be expressed as a sum over the contribution from any object which lies

5 This states that a vector joining two masses will sweep out equal areas in equal times, which is equivalent to the conservation of the specific angular momentum, $h=r^{2} \dot{f}$. 
along the line-of-sight,

$$
\begin{aligned}
\delta I_{\nu}(\hat{\boldsymbol{n}})= & \frac{1}{N} \sum_{i=1}^{N} \theta^{(2)}\left(R_{i} / r_{i}-\left|\hat{\boldsymbol{n}}-\hat{n}_{i}\right|\right) \\
& \left.\times\left[B_{\nu}\left(T_{\text {Oort }}^{i}\right)\right)-B_{\nu}\left(\bar{T}_{\mathrm{cmb}}\right)\right]
\end{aligned}
$$

here $\hat{n}_{i}$ is the position on the sky of the Oort Cloud object's centroid and the $R_{i}$ is the Oort Cloud object's radius and $r_{i}$ its radial distance. The $\theta$ function simply requires that the direction of interest intersects the relevant Oort Cloud object. Here $\mathrm{N}$ is the total number of objects in the sample that we are considering. We can directly calculate the Poisson fluctuations

$$
\begin{aligned}
\left\langle\delta I_{\nu}\left(\hat{\boldsymbol{n}}_{1}\right)\right. & \left.\delta I_{\nu}\left(\hat{\boldsymbol{n}}_{2}\right)\right\rangle=\frac{1}{N^{2}} \sum_{i=1}^{N}\left\langle\theta^{(2)}\left(R_{i} / D_{i}-\left|\hat{\boldsymbol{n}}_{1}-\hat{n}_{i}\right|\right)\right. \\
& \left.\times \theta^{(2)}\left(R_{i} / D_{i}-\left|\hat{\boldsymbol{n}}_{2}-\hat{n}_{i}\right|\right)\left[B_{\nu}\left(T_{\text {Oort }}^{i}\right)-B_{\nu}\left(\bar{T}_{\mathrm{cmb}}\right)\right]^{2}\right\rangle .
\end{aligned}
$$

The Oort Cloud objects subtend a finite angle on the sky and Poisson fluctuations will cause CMB fluctuations if $\hat{\boldsymbol{n}}_{1}$ and $\hat{\boldsymbol{n}}_{2}$ intersect the same object. The expectation value is taken over the assumed distribution of orbital elements and masses.

We are interested in the intensity fluctuation averaged over some angular aperature, which we take to be a top-hat of angular size $\bar{\theta}$,

$$
\bar{\delta} I_{\theta}=\int \frac{d^{2} \hat{\boldsymbol{n}}}{\bar{\theta}^{2}} \delta I_{\nu}(\hat{\boldsymbol{n}})
$$

The power induced in the CMB by the Poisson fluctuations can be related the variance of the aperature averaged intensity fluctuations as

$$
\frac{\ell^{2} C_{\ell}}{2 \pi}=\left\langle\left(\frac{\overline{\delta I}_{\nu}}{B_{\nu}\left(\bar{T}_{\mathrm{cmb}}\right)}\right)^{2}\right\rangle
$$

where smoothing was done on the angular scale $\theta=2 \pi / \ell$.

At a fixed time the average over the initial time of perihelon is equivalent to performing a time average over the orbital period which can be related to an average over the true anomaly. After we perform these averages we find that the power spectrum produced by Poisson fluctuations is

$$
\begin{aligned}
C_{\ell}= & \frac{1}{2 \pi} \frac{1}{N} \int d M d a d e d \Theta P(\Theta) P(a) P(e) P(M) \\
& \times \pi\left(\frac{R}{D}\right)^{2}\left[\frac{B_{\nu}\left(T_{\text {Oort }}(D)\right)}{B_{\nu}\left(\bar{T}_{\mathrm{cmb}}\right)}-1\right]^{2} .
\end{aligned}
$$

The distributions of orbital elements and mass are given in Eqs. 23 \& 7 , respectively. The integrals over the three orientation angles are unity. Although 


\begin{tabular}{ccc}
\hline \hline \multicolumn{1}{c}{$C_{2}^{\text {OC }}$} & $C_{2}^{\text {code }}$ \\
\hline \hline $30 \mathrm{GHz}$ & $1.3 \times 10^{-32}$ & $1.3 \times 10^{-14}$ \\
$100 \mathrm{GHz}$ & $5.2 \times 10^{-32}$ & $5.2 \times 10^{-14}$ \\
$217 \mathrm{GHz}$ & $7.4 \times 10^{-31}$ & $7.4 \times 10^{-13}$ \\
$353 \mathrm{GHz}$ & $2.5 \times 10^{-29}$ & $2.5 \times 10^{-11}$ \\
$545 \mathrm{GHz}$ & $5.4 \times 10^{-27}$ & $5.4 \times 10^{-9}$ \\
\hline \hline
\end{tabular}

Table 2

Fractional Poisson fluctuations in the number of Oort Cloud objects at different frequencies. The fractional fluctuation relevant for the calculations of our code $\left(C_{2}^{\text {code }}\right)$ and the real Oort Cloud $\left(C_{2}^{\mathrm{OC}}\right)$ are shown.

we will apply this formula to the quadrupole $(\ell=2)$, it is applicable to the power spectrum on any angular scale.

There are two distinct reasons that we are interested in Poisson fluctuations. The first involves understanding the accuracy of our numerical calculations, described in $\$ 2.3$. We will perform our calculation with $10^{3}$ realizations of $10^{6}$ test particles, but due to the stronger weighting of the test particles closest to perihelia, the effective number of test particles is reduced and the fractional fluctuation amplitude increased. The second is related to the statistical significance of any observed CMB spectral distortion. As Oort Cloud objects move along their orbits the closest objects, and therefore the objects that produce the largest signal, will change. These Poisson fluctuations could potentially limit the statistical significance and therefore usefulness of any measurement of CMB spectral distortions. The results of our calculations are shown in Table 2 for the fractional fluctuations relevant to both our code and the real Oort Cloud.

We conclude that statistical fluctuations will not be important when interpreting the observational data if our model of the size distribution of Oort Cloud objects is reasonable. These fluctuations will be much smaller than standard experimental noise. However, the statistical fluctuations present in our numerical calculations, which contain significantly fewer test particles than the true number of Oort Cloud objects, will be considerably larger and comparable to the magnitude of the effect that we are interested in. Therefore we will calculate the distribution of quadrupole moments by calculating the quadrupole at $10^{3}$ different times. From this distribution we can estimate the magnitude of the perturbation on the shape of the Oort Cloud, as well as, the effect of Poisson fluctuations on the resultant CMB spectral distortions. 


\subsection{Initial Conditions}

The comets in the Oort Cloud, in the absense of external perturbations, effectively act as test particles that move on Keplerian orbits around the Sun. The hypothetical stellar encounter simply perturbs the comets onto Keplerian orbits with new orbital element: 6 . The orbits are completely described by six orbital elements - (i) the semi-major axis (a); (ii) the eccentricity (e); (iii) the inclination of the orbital plane $(i)$; (iv) the longitude of perihelion $(\omega)$; (v) the longitude of the ascending node $(\Omega)$; and (vi) the time of perihelion 7 $(\Theta)$. The inclination angle, the longitude of perihelion and the longitude of the ascending node, are rotation angles used to convert the coordinate system of invariant plane (which is different for every comet) to a standard reference coordinate system. With these orbital elements the motion of the comet around the Sun is completely specified.

We set the initial conditions of the Oort Cloud objects by randomly sampling the uniform distributions

$$
\begin{aligned}
0.3 & \leq e \leq 1-\frac{50 \mathrm{AU}}{a} \\
-1 & \leq \cos I \leq 1 \\
0 & \leq \omega \leq 2 \pi \\
0 & \leq \Omega \leq 2 \pi \\
0 & \leq \Theta \leq P
\end{aligned}
$$

where $P=2 \pi a^{3 / 2} /\left(G M_{\odot}\right)^{1 / 2}$ is the test particle's orbital period. The maximum eccentricity is determined by the condition that the comet's perihelion lies far enough away from Neptune that it becomes dynamically decoupled. This condition roughly requires $r_{p}=a(1-e) \geq 50 \mathrm{AU}$, so the maximum eccentricity depends on on the semi-major axis of the orbit. Simulations of the formation of the Oort Cloud have found that the probability distribution of semi-major axes has a power-law form $P(a) \propto a^{-\gamma}$, but have disagreed on the value of its index $\gamma$. Originally it was stated by Duncan et al. (1987) that $\gamma \sim 3.5$, but updated simulations with more realistic initial conditions for the comets have found a shallower value, $\gamma \sim 1.5$ (Dones et al., 2007). When we present numerical results in $\$ 4$ we adopt the value of $\gamma=3$. We will assume that the inner edge of the Oort Cloud is $a=10^{3} \mathrm{AU}$. We take the inner edge to be semi-major axis beyond which the Oort Cloud becomes roughly spherical. The Scattered Disc lies inside of this radius. And we pick the outer edge to lie at $a=10^{4} \mathrm{AU}$. We then randomly choose an initial time $0 \leq t_{i} \leq P$

\footnotetext{
${ }_{6}$ We also do allow the comets to be ejected from the Solar System if the final eccentricity if greater than one.

7 Ordinarily $\tau$ is used to designate the time of perihelion passage, we chose a different symbol to prevent confusion with the use of $\tau$ for the optical depth.
} 
to calculate the initial position in order to evaluate the above perturbation equations. Then we choose another random final time $0 \leq t_{f} \leq P$ in order to evaluate the final positions of the comets.

The initial conditions of the Oort Cloud before the stellar encounter depend on the rate at which the comets are scattered to high inclination angles 8 . At smaller distances, the isotropization processes are less efficient and the cloud will be smoothly compressed into a torus that extends out of the ecliptic plane. The initial conditions depend on the efficiency of these relaxation processes. These processes include the effects from passing stars and molecular clouds, the tides of the Galactic disc, and the potential of the parent molecular cloud inside of which the Sun had formed. Placing a large amount of mass into the nonspherical Scattered Disc or any primordial asphericity in the Oort cloud will only increase the anisotropic spectral distortions. The intensity fluctuations induced by the Scattered Disc estimated in this paper can be viewed as a lower limit for the signal.

We estimate of the influence of the initial conditions by calculating the expected quadrupole signal from the Scattered Disc. We assume that the total mass is $0.1 M_{\oplus}$ and the semi-major axes lie between $10^{2} \mathrm{AU}$ and $10^{3} \mathrm{AU}$. Most importantly we choose the disc's opening angle to be 30 degrees. The six quadrupole moments at three frequencies, $\nu=30 \mathrm{GHz}$ (black), $\nu=143 \mathrm{GHz}$ (red) and $\nu=217 \mathrm{GHz}$ (blue), are shown in Fig. 11, The mixed quadrupole moments, such as $Q_{x y}$, all vanish within Poisson fluctuations. As expected Poisson fluctuations become larger at higher frequencies. The diagonal quadrupole moments, such as $Q_{x x}$, do not vanish. The $Q_{z z}$ moment does not vanish because the disc is compressed into the Ecliptic Plane. The traceless condition, $Q_{x x}+Q_{y y}+Q_{z z}=0$, forces the other two diagonal moments to be non-zero. Since the scattered disc is azimuthally symmetric by construction, $Q_{x x}$ and $Q_{y y}$ are forced to be equal.

\subsection{Orbital Perturbation}

The external perturbation from a passing star acts both on the Oort cloud as well as the Sun which are bound together, and so only the relative (tidal) acceleration between the comet and the Sun is relevant

$$
\begin{aligned}
\Delta \boldsymbol{a} \equiv & \boldsymbol{a}_{\text {Oort }}-\boldsymbol{a}_{\odot}, \\
& =\frac{G M_{p}(\boldsymbol{b}-\boldsymbol{r})}{|\boldsymbol{b}-\boldsymbol{r}|^{3}}-\frac{G M_{p} \boldsymbol{b}}{b^{3}},
\end{aligned}
$$

8 The initial distribution of $\omega$ and $\Omega$ should have been roughly uniform even in the proto-planetary disc. 

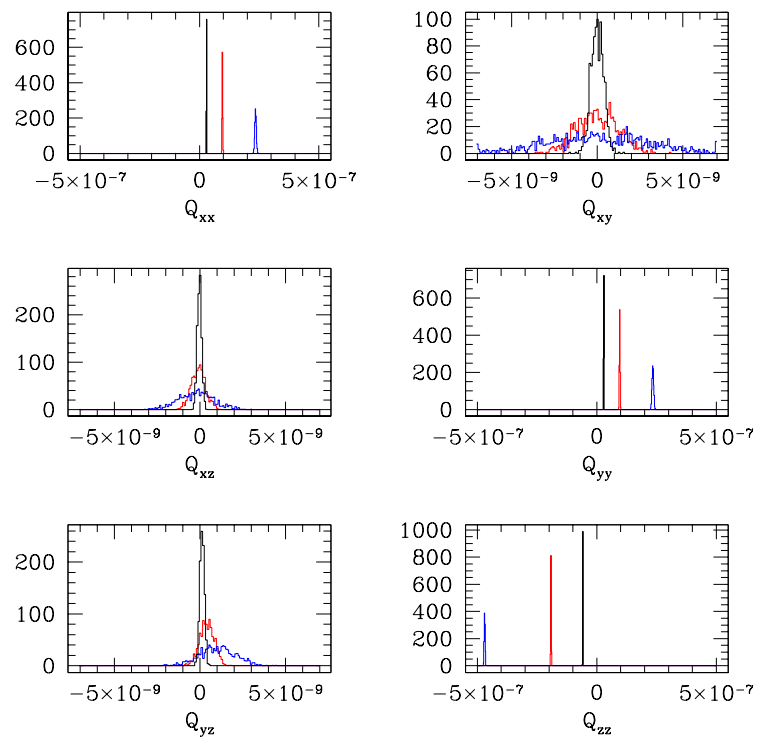

Fig. 1. Histograms of the six quadrupole moments at three different frequencies $\nu=30 \mathrm{GHz}$ (black), $\nu=143 \mathrm{GHz}$ (red) and $\nu=217 \mathrm{GHz}$ (blue). The mixed quadrupole moments all vanish within Poisson fluctuations and the diagonal moments have are nonzero at a statistically significant level.

where $M_{p}$ is the mass of the perturber and $\boldsymbol{b}$ is the vector from the Sun to the perturber.

Kepler's third law, $T \propto a^{3 / 2}$, implies that an object on an orbit with $a=$ $1000 \mathrm{AU}$ has an orbital period, $T=10^{12} \mathrm{~s}$. If the impact parameter of the perturber is $b \approx 2000 \mathrm{AU}=0.01 \mathrm{pc}$ and it is traveling at a speed of $v=30 \mathrm{~km} / \mathrm{s}$, then the effective duration of the impulse would be $T_{\text {pert }} \lesssim 2 b / v=2 \times 10^{10} \mathrm{~s}$. The orbital period is typically longer than the duration of the impulse, and so we will use the impulse approximation for simplicity. Numerical experiments have found that the validity of the impulse approximation even extends to the regime where $T \approx 0.2 \times T_{\text {pert }}$ (Aguilar \& White, 1985). For test particles with larger semi-major axes the accuracy of the impulse approximation becomes increasingly better.

Clearly, a passage through the Oort Cloud would strongly perturb the comets that lie nearly along the perturber's trajectory (Weissman, 1990). Such an effect is believed to be responsible for the comet showers that occasionally take place (as the strongly perturbed comets either enter the inner Solar System or get ejected from the Oort Cloud), but will these strongly perturbed Oort Cloud objects will have little effect on results because they are assumed to have been removed from the Oort Cloud. In our calculation will assume that all test particles that are more strongly attracted to the perturber than the Sun are ejected. Initially this has a large effect on the angular structure of the Oort Cloud, however the effect is quickly diminished due to phase mixing. After a 
test particle is ejected from the Solar System a hole is left in the Oort Cloud that orbits around the sun with the orbital parameters of the original test particle. This results in an underdensity of test particles that would produce anisotropy in the same manner as an overdensity would. However the large spread in orbital periods, due to the large spread in semi-major axes, means that the signal vanishes over a timescale corresponding to the orbital period of the test particles. Of course, the mean signal will remain the same as this only depends on the total covering fraction of Oort Cloud objects. However the quadrupole, or any angular moments, will vanish as the holes left by the ejected test particles lose spatial coherence.

In the impulse approximation, only the component of the force perpendicular to the perturber's motion remains after its path is integrated over (Binney \& Tremaine, 1987). Therefore we must apply the projection operator

$$
T=I-\hat{v} \hat{v}
$$

to the tidal force, where $\boldsymbol{v}$ is the velocity of the perturber. The components of the projected tidal force, written in a coordinate basis attached to the comet, are

$$
\begin{aligned}
& R=\hat{\boldsymbol{e}}_{R} \cdot \boldsymbol{T} \cdot \Delta \boldsymbol{a}, \\
& T=\hat{\boldsymbol{e}}_{T} \cdot \boldsymbol{T} \cdot \Delta \boldsymbol{a}, \\
& N=\hat{\boldsymbol{e}}_{N} \cdot \boldsymbol{T} \cdot \Delta \boldsymbol{a},
\end{aligned}
$$

here $\hat{\boldsymbol{e}}_{R}$ is the radial basis vector, $\hat{\boldsymbol{e}}_{T}$ is the tangential basis vector and $\hat{\boldsymbol{e}}_{N}$ is the normal basis vector. The Lagrange equations describe how the perturbation 
changes the orbital elements (Danby, 1962; Murray \& Dermott, 2000)

$$
\begin{aligned}
\frac{d a}{d t}= & \frac{2 a^{3 / 2}}{\sqrt{\mu\left(1-e^{2}\right)}}[R e \sin f+T(1+e \sin f)] \\
\frac{d e}{d t}= & \sqrt{\frac{a\left(1-e^{2}\right)}{\mu}}\left[R \sin f+T\left(\cos f+\frac{e+\cos f}{1+e \cos f}\right)\right] \\
\frac{d i}{d t}= & \sqrt{\frac{a\left(1-e^{2}\right)}{\mu}} \frac{N \cos (\omega+f)}{1+e \cos f} \\
\frac{d \Omega}{d t}= & \sqrt{\frac{a\left(1-e^{2}\right)}{\mu}} \frac{N \sin (\omega+f)}{\sin i(1+e \cos f)} \\
\frac{d \omega}{d t}= & \sqrt{\frac{a\left(1-e^{2}\right)}{e^{2} \mu}}\left[-R \cos f+T \sin f \frac{2+e \cos f}{1+e \cos f}\right] \\
\frac{d \Theta}{d t}= & 3 R(\Theta-t) \sqrt{\frac{d \Omega}{d t} \cos i,} e \sin f \\
& +R \frac{a^{2}\left(1-e^{2}\right)}{\mu}\left(\frac{2}{1+e \cos f}-\frac{\cos f}{e}\right) \\
+ & 3 T(\Theta-t) \sqrt{\frac{a}{\mu\left(1-e^{2}\right)}} e \sin f \\
& +T \frac{a^{2}\left(1-e^{2}\right) \frac{\sin f(2+e \cos f)}{e \mu}}{1+e \cos f}
\end{aligned}
$$

where $\mu=G M_{\odot}$.

Within the impulse approximation, we assume that the internal configuration of the solar system is frozen during the close encounter with the perturber; it is this assumption that causes the force parallel to the perturber's velocity to exact cancel. Therefore the change in the orbital element, $\mathrm{x}$, can be expressed as

$$
\Delta x \approx \frac{d x}{d t} \frac{2 b}{v}
$$

The new distribution of orbital elements will be solely due to the addition of velocity perturbations, because all previous comets with the given elements will have been perturbed to other orbits

$$
p\left(\left\{x_{i}\right\}\right)=\int p_{0}\left(x_{i}-\Delta x_{i}\right) D\left(\Delta x_{i}\right)
$$

In the test particle limit, the mass of the comet should not affect how it is perturbed by the stellar companion. Therefore the distribution function of the Oort Could comets should be factorizable into the distribution of comet 
orbital elements and the mass function of comets

$$
f_{\text {Oort }}=\frac{d n}{d M}(M) \times p\left(\left\{x_{i}\right\}\right) .
$$

We will use the orbital element distribution function in Eq. (41) to calculate the CMB intensity fluctuation using Eq. (5). In principle the orbital element distribution function can be used to construct a distribution function parameterized by the Keplerian integrals of motion. Since the distribution function is six dimensional and we are interested in observable signatures in the CMB, we will not focus on this aspect of the problem. However we will mention a condition that the distribution function must satisfied if an angular signal will be produced. For a quadrupole, or any angular moment, intensity fluctuation to be non-zero, the distribution of Euler angles $(\Omega, \omega, i)$ must be correlated with the distribution of semi-major axes and eccentricities. In our calculations we initially assume that all the orbital elements are uncorrelated and that the Oort Cloud is statistically isotropic. The stellar perturbation induces the necessary correlations to produce a statistically significant signal.

\subsection{Likely Encounter Parameters}

In addition to determining the initial conditions of the Oort Cloud objects we need to specify the properties of the perturber. Observations of local stars can identify their impact parameter with respect to the Solar System only up to 10 Myr in the past (García-Sánchez et al., 1999, 2001). Of course, we can use the initial mass function of stars and their velocity dispersion with respect to the local standard of rest to make statistical statements about the likely properties of the perturber, however for a single perturber that gets to the closest approach and has the biggest effect, such statistical statements are not very useful. In $\$ 5$ we will discuss a Bayesian method that allow us to use a detected signal to statistically constrain the properties of the perturber. Given a number density and a relative velocity with respect to the Sun the minimum impact parameter of the perturber, $R_{\text {min }}$, can be estimated by requiring that the probability of encounter during some time period, $\Delta t$, is of order unity

$$
P=\pi R^{2} n_{\mathrm{stars}} v \Delta t \approx 1
$$

The environment of the Solar System might have been quite different in the past than it is today. As mentioned in \$1, the Sun might have formed in a stellar cluster and so the number density of neighboring stars may have been much higher. Also as the Solar System undergoes epicyclic and vertical oscillations with respect to the Galactic disc or passes through spiral arms, the 


\begin{tabular}{ccccc}
\hline \hline Model & $n\left(\mathrm{pc}^{-3}\right)$ & $v_{\text {rel }}\left(\mathrm{km} \mathrm{s}^{-1}\right)$ & $\Delta t(\mathrm{Gyr})$ & $R_{\min }(\mathrm{AU})$ \\
\hline \hline Present Neighborhood - A & 0.1 & 30 & 4 & 1100 \\
Present Neighborhood - B & 0.1 & 30 & 1 & 2100 \\
Present Neighborhood - C & 0.1 & 30 & 0.5 & 3000 \\
Cluster - A & 1000 & 1 & 0.01 & 1100 \\
Cluster - B & 100 & 1 & 0.01 & 3700 \\
\hline \hline
\end{tabular}

Table 3

Characteristic number densities, velocities, time intervals and minimum impact parameters for several models of the local Solar neighborhood. We adopt the parameters of 'Present Neighborhood - B' for our calculations.

environment also changes. The closest encounter probabilities can be adjusted to account for the changing Solar environment.

A variety of relaxation processes - passing stars and giant molecular clouds (Duncan et al., 1987; Hut \& Tremaine, 1985), precession due to the potential of the Galactic disc (Heisler \& Tremaine, 1986) and the self gravity of the Oort Cloud (Tremaine, 2005) - can isotropize the orbits of objects in Oort Cloud and reduce the non-sphericity produced by the stellar encounter. Of course the possibility of eliminating these signatures does not imply that the Oort Cloud will be spherical today, but that it will possess the non-sphericity induced by the closest stellar encounter that occurred at a time less than the appropriate relaxation timescale. The self-gravity of the Oort Cloud was determined to produce the largest effect; the calculated timescale was $1 \mathrm{Gyr}$ (Tremaine, 2005). More calculations are needed to determine the effect on nearly parabolic orbits, which spend considerable amounts of time at different distances and therefore experience different gravitational perturbations. We will leave a detailed analysis of these relaxation processes to future work. Therefore we will consider the minimum impact parameters for the current Solar neighborhood with a variety of time intervals $-\Delta t=0.5,1,4 \mathrm{Gyr}$. These models are described in Table 3 .

Of course rare events $(P \ll 1)$ sometimes occur and the above figures should be seen as estimates of the mean of the distribution from which the actual closest impact parameter has been randomly drawn. The probability that the closest encounter occurs with impact parameter $R$ is equivalent to the joint probability that one encounter occurred at $R, P(1 \mid R)$, and no encounters 


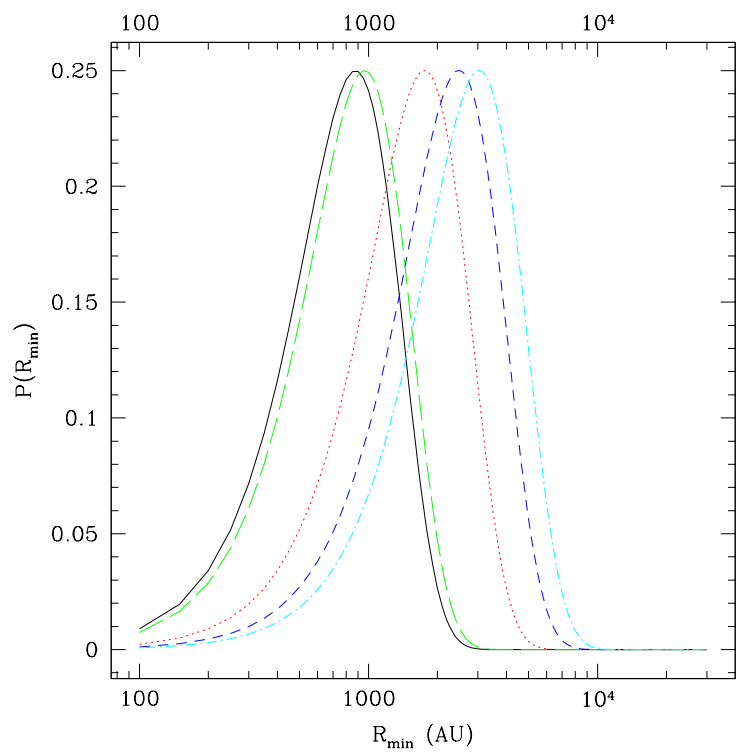

Fig. 2. Plot of the probability distribution of minimum impact parameters for the models described in Table (3). The curves in a left-to-right manner correspond to Neighborhood-A (black, solid curve), Cluster-A (green, long dashed curve), Neighborhood-B (red, dotted curve), Neighborhood-C (blue, dashed curve) and Cluster-B (cyan, dot-dashed curve). We adopt the Neighborhood-B model for calculations in this paper.

occurred with $r<R, P(0 \mid r<R)$,

$$
\begin{aligned}
P\left(R_{\text {min }}\right) & =P(0 \mid r<R) \times P(1 \mid R), \\
& =\int_{0}^{R} p(r) d r e^{-\pi r^{2} n v \Delta t} \times\left(\pi R^{2} n v \Delta t\right) e^{-\pi R^{2} n v \Delta t}, \\
& =e^{-\pi R^{2} n v \Delta t}\left(1-e^{-\pi R^{2} n v \Delta t}\right) .
\end{aligned}
$$

This probability has a maximum at $P=\ln 2 \sim 0.7$, instead of $P=1$ in Eq. (42). The probability distribution of $R_{\min }$ for the variety of environments are shown in Fig. 2 .

\section{Analytic Estimates}

Before we present numerical results in $\$ 4$ it is worthwhile to look at orderof-magnitude estimates of the effect. Using the impulse approximation it is straightforward to determine how the perturbation affects the test particles. The velocity and kinetic energy are directly changed by the impulse, but the potential energy remains the same since the distance from the Sun has not changed. If the system was self-gravitating and the traditional virial theorem applicable, the system would relax to a new equilibrium distribution and 
the moments of the gravitational potential tensor would directly reflect the changed velocity dispersion tensor (Binney \& Tremaine, 1987). Relating the velocity or energy kick to a quadrupole moment requires a generalization of the commonly used virial theorem as we show below.

\subsection{Virial Equations}

The ensemble of test particles in the Oort Cloud satisfies the collisionless Boltzmann equation

$$
\frac{\partial f}{\partial t}+\boldsymbol{v} \cdot \nabla f-\nabla \Phi \cdot \nabla_{v} f=0
$$

where the distribution function depends on position, velocity and time, although we will suppress this functional dependence for brevity. We will assume that the Oort Cloud is not self-gravitating, so the potential through which the test particles are moving is solely determined by the Sun's gravity

$$
\Phi(\boldsymbol{x})=-\frac{G M_{\odot}}{r} .
$$

Assuming the distribution is time-independent, which is equivalent to assuming that Poisson fluctuations in the final moments are negligible (see \$2.1), we find the constraint equation

$$
G M_{\odot} \frac{\boldsymbol{x} \cdot \nabla_{v} f}{r^{3}}=\boldsymbol{v} \cdot \nabla f
$$

In simple words this equation states that the number of test particles advected from a phase space volume element equals the number of test particles accelerated into this volume element by the Sun's gravity. If we were deriving the traditional virial theorem we would take velocity and position moments of this equation or the time-dependent version of it, Eq. (44). However we are interested in relating the velocity dispersion tensor to the intensity quadrupole moments, not the potential energy tensor.

The quadrupole moment can be expressed as an integral over the Oort Cloud volume

$$
\begin{aligned}
Q_{i j}(\nu) B_{\nu}\left(T_{\mathrm{CMB}}\right)= & \int d^{3} \boldsymbol{r} d^{3} \boldsymbol{v} f \frac{f_{A}}{r^{4}}\left(x_{i} x_{j}-\frac{1}{3} r^{2} \delta_{i j}\right) \\
& \times\left[B_{\nu}\left(T_{\text {Oort }}\right)-B_{\nu}\left(T_{\mathrm{CMB}}\right)\right]
\end{aligned}
$$

The optical depth and the distribution function are defined such that

$$
\int d^{3} \boldsymbol{r} d^{3} \boldsymbol{v} f(\boldsymbol{x}, \boldsymbol{v})=1
$$


The blackbody frequency spectrum is in general a complex function of $T_{\text {Oort }}$, although at low frequencies it scales as $B_{\nu}\left(T_{\text {Oort }}\right) \propto T_{\text {Oort }}$. In order to derive analytic results we will express the blackbody spectrum's dependence on distance as a power law,

$$
B_{\nu}\left(T_{\text {Oort }}\right)=\frac{A_{\nu}}{r^{\beta}},
$$

where the exponent is defined as

$$
\beta=\frac{1}{2} \frac{\partial \ln B_{\nu}(T)}{\partial \ln T} .
$$

This exponent is only well defined in Rayleigh-Jean's limit where it equals a constant, $\beta=1 / 2$. At higher frequencies its value grows rapidly and is strongly frequency dependent. Below we will use $\beta=1 / 2$, but comment on how the higher frequency cases will affect our results.

We first multiply Eq. (46) by $\boldsymbol{v} \boldsymbol{x} r^{-(1+\beta)}$. Now following the basic steps in deriving the virial theorem we will integrate this equation over phase space and then integrate by parts to eliminate the position and velocity derivatives acting on the distribution function. This will allow us to relate the first part, the $x_{i} x_{j}$ term, of the quadrupole moment to the velocity dispersion tensor. The second part, the $-\delta_{i j} / 3$ term, can be related to the velocity through the energy equation of a Keplerian orbit

$$
E=\frac{v^{2}}{2}-\frac{G M_{\odot}}{r}=-\frac{G M_{\odot}}{2 a} .
$$

For simplicity, here and in the next subsection we assume zero eccentricity orbits with $v^{2}=G M_{\odot} / a$.

The final equation relating the quadrupole moments to the velocity distribution of the test particles is

$$
\begin{aligned}
& Q_{i j}(\nu) B_{\nu}\left(T_{\mathrm{CMB}}\right)=f_{A} \int d^{3} \boldsymbol{r} d^{3} \boldsymbol{v} f(\boldsymbol{x}, \boldsymbol{v})\left\{A_{\nu}\right. \\
& \times\left[\frac{v_{i} v_{j}}{G M_{\odot} r^{1+\beta}}-\frac{(2+\beta)\left(x_{i} v_{j}+v_{i} x_{j}\right)(\boldsymbol{x} \cdot \boldsymbol{v})}{2 G M_{\odot} r^{3+\beta}}-\frac{\delta_{i j}}{3}\left(\frac{v^{2}}{G M_{\odot}}\right)^{2+\beta}\right] \\
& \left.-B_{\nu}\left(T_{\mathrm{CMB}}\right)\left[\frac{v_{i} v_{j}}{G M_{\odot} r}-\frac{\left(x_{i} v_{j}+v_{i} x_{j}\right)(\boldsymbol{x} \cdot \boldsymbol{v})}{G M_{\odot} r^{3}}-\frac{\delta_{i j}}{3}\left(\frac{v^{2}}{G M_{\odot}}\right)^{2}\right]\right\} .
\end{aligned}
$$

\subsection{Perturbations}

In this subsection we will make several approximations that will allow us to calculate analytic expressions for the quadrupole moments. We will adopt 
the impulse approximation as well as the tidal approximation for the relative acceleration of the test particles with respect to the Sun

$$
\Delta \boldsymbol{a}_{r e l}=\frac{G M_{p}}{b^{3}}[\boldsymbol{r}-3 \hat{\boldsymbol{b}}(\boldsymbol{r} \cdot \hat{\boldsymbol{b}})] .
$$

In this expression for the relative acceleration, $M_{p}$ is the perturber mass, $\boldsymbol{r}$ is the radial vector between the Sun and the Oort Cloud object, and $\boldsymbol{b}$ is the impact parameter vector between the Sun and the perturber. We are able to use the tidal approximation in this section because we will assume a thin shell distribution of semi-major axes for all the test-particles. In our numerical calculations we assume a power-law distribution of semi-major axes between $a=10^{3} \mathrm{AU}$ and $10^{4} \mathrm{AU}$. With this latter distribution our analytic model assumption of the tidal acceleration is invalid because for the appropriate values of $R_{\min }$ the perturber passes through the Oort Cloud.

With these assumptions for the analytic model, the velocity kick is,

$$
\Delta \boldsymbol{v}=\frac{2 G M_{p}}{B^{2} v_{r e l}}\left(\boldsymbol{I}-\hat{\boldsymbol{v}}^{p} \hat{\boldsymbol{v}}^{p}\right)[\boldsymbol{r}-3 \hat{\boldsymbol{b}}(\boldsymbol{r} \cdot \hat{\boldsymbol{b}})],
$$

where we project out the component of the force parallel to the perturber's trajectory. For later convenience we will define

$$
\Psi=\hat{\boldsymbol{n}}-3 \hat{\boldsymbol{b}}(\hat{\boldsymbol{n}} \cdot \hat{\boldsymbol{b}})-\hat{\boldsymbol{v}}^{p}\left(\hat{\boldsymbol{n}} \cdot \hat{\boldsymbol{v}}^{p}\right),
$$

such that

$$
\Delta \boldsymbol{v}=\frac{2 G M_{p} r}{b^{2} v_{r e l}} \boldsymbol{\Psi}
$$

Here we have assumed

$$
\hat{\boldsymbol{v}}_{r e l} \cdot \hat{\boldsymbol{b}}=0
$$

which directly follows from our use of the impulse approximation. Working within the impulse approximation the direction of the impact parameter is almost entirely determined by the direction of the encounter velocity. This constraint from the impulse approximation allows us to simplify the form of the above velocity kick. There is still phase information that is needed to completely specify the geometry, Eq. (57) is simply one equation for two unknowns. For example, the direction of the encounter only determines a plane within which the direction of the encounter velocity must lie; the azimuthal angle of the encounter velocity must still be specified. Equivalently, the direction of the encounter velocity determines a great circle on the sky along which the encounter must have occured; the longitudinal position along that great circle must still be specified. The azimuthal angle of the relative velocity should have uniform distributions between 0 and $\pi$ (recall the $\hat{\boldsymbol{v}} \rightarrow-\hat{\boldsymbol{v}}$ degeneracy mentioned above), whereas the longitudal angle that determines the direction of the impact parameter should be uniformly distributed between 0 and $2 \pi$. 
There is no change in the velocity dispersion, or the quadrupole moments, at first order in $\Delta \boldsymbol{v}$. This is a consequence of the fact that we assumed the Oort Cloud to be statistically time-independent and axisymmetric. At second order the velocity kick will produce a non-zero quadrupole moment,

$$
\begin{aligned}
& Q_{i j}(\nu) B_{\nu}\left(T_{\mathrm{CMB}}\right)=\frac{4 G M_{p}^{2} \tau a^{3}}{b^{4} v_{\mathrm{vel}}^{2} M_{\odot}} \int d^{2} \hat{\boldsymbol{n}}\left\{\frac { A _ { \nu } } { a ^ { \beta } } \left[\Psi_{i} \Psi_{j}\right.\right. \\
& \left.-\left(1+\frac{\beta}{2}\right)\left(\Psi_{i} n_{j}+\Psi_{j} n_{i}\right)(\hat{\boldsymbol{n}} \cdot \Psi)-\frac{(2+\beta)}{3} \delta_{i j} \Psi \cdot \Psi\right] \\
& \left.-B_{\nu}\left(T_{\mathrm{CMB}}\right)\left[\Psi_{i} \Psi_{j}-\left(\Psi_{i} n_{j}+\Psi_{j} n_{i}\right)(\hat{\boldsymbol{n}} \cdot \Psi)-\frac{2}{3} \delta_{i j} \Psi \cdot \Psi\right]\right\}
\end{aligned}
$$

where we have assumed that the Oort Cloud is made of a thin shell of zero eccentricity test particles all with semi-major axis $a$. Since we assume that all of the Oort Cloud objects lie at a single semi-major axis $a$ we can replace the absolute covering fraction $f_{A}$, defined in Eq. 6, with the optical depth $\tau$.

The quadrupole tensor can now be directly evaluated as

$$
Q_{i j}(\nu)=\frac{16 \pi G M_{p}^{2} \tau a^{3}}{15 b^{4} v_{\mathrm{vel}}^{2}}\left[\frac{B_{\nu}\left(T_{\mathrm{Oort}}\right)}{B_{\nu}\left(T_{\mathrm{CMB}}\right)} A_{i j}(\beta)-A_{i j}(0)\right],
$$

where the configuration tensor is defined as

$$
A_{i j}(\beta)=\left[(3-\beta) v_{i} v_{j}+3(1+3 \beta) b_{i} b_{j}\right]+\frac{41+28 \beta}{3} \delta_{i j}
$$

Note that $v_{i}$ and $b_{i}$ are components of $\hat{\boldsymbol{b}}$ and $\hat{\boldsymbol{v}}^{p}$, which have unit norm.

Taking some characteristic numbers, such as $M_{p}=1 M_{\odot}, \beta=1 / 2,9 v_{\text {rel }}=$ $30 \mathrm{~km} \mathrm{~s}^{-1}, a=10^{3} \mathrm{AU}$ and $b=2 \times 10^{3} \mathrm{AU}$, we find

$$
Q(\nu)=3.9 \times 10^{-9}\left(\frac{\tau}{10^{-6}}\right)\left[\frac{B_{\nu}(10 \mathrm{~K})}{B_{\nu}(3 \mathrm{~K})}-1\right]
$$

Ignoring the directional dependence described above, the amplitude of $Q$ varies from $1.4 \times 10^{-8}$ at low frequency to $2.3 \times 10^{-7}$ at $\nu=300 \mathrm{GHz}$ and $8.9 \times 10^{-6}$ at $\nu=600 \mathrm{GHz}$. Our choice of $\tau=10^{-6}$ is close to the value of our adopted model in Table 1. Since we have assumed that initial distributions of the test particle sizes and orbital elements are independent, we can simply scale the value of $Q(\nu)$ to the appropriate value of $\tau$.

$\overline{9}$ For simplicity we choose to evaluate the configuration tensor at $\beta=1 / 2$ for all frequencies despite the fact that $\beta$ and therefore $Q$ will grow at higher frequencies. 

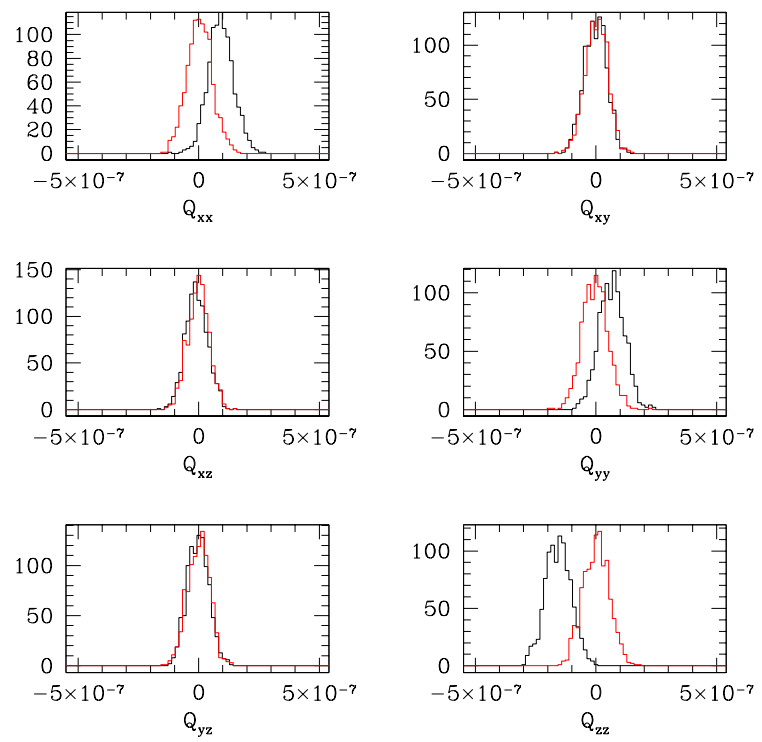

Fig. 3. Histogram of the induced CMB quadrupoles at $\nu=30 \mathrm{GHz}$ before (red curve) and after (black curve) the perturbation. The quadrupole moments are in units of mean CMB intensity.

\section{Numerical Results}

Next we present our numerical results. We start by randomly choosing the initial orbital elements of $10^{6}$ Oort Cloud objects according to the distributions in Eqs. (23). Then for a given trajectory and mass of the external perturber, we use Eqs. (33) to map the initial orbital elements to the final orbital elements. The resulting quadrupole moments, calculated using Eq. (14), are determined for $10^{3}$ distinct initial and final times in order to estimate the effect of statistical fluctuations on the outcome and therefore determine if the perturbation produced a statistically significant change in the structure of the Oort Cloud.

These quadrupole distributions are shown in Fig. 3 for $\nu=30 \mathrm{GHz}$, Fig. 4 for $\nu=353 \mathrm{GHz}$ and Fig. 5 for $\nu=545 \mathrm{GHz}$. We choose these frequencies to correspond to the frequency bins of the Planck satellite. The statistical fluctuations are greater at high frequency because the induced signal scales as a stronger power of radial distance, as seen in Eq. (15). For this particular example the direction of impact parameter was $\hat{\boldsymbol{B}}=(0.909,0,-0.416)$ and the trajectory of the perturber was $\hat{\boldsymbol{v}}_{p}=(0,1,0)$. The perturber's mass was $M_{p}=1 M_{\odot}$, its relative velocity $v_{p}=30 \mathrm{~km} \mathrm{~s}^{-1}$ and impact parameter $b=$ $2100 \mathrm{AU}$.

From the distributions of the quadrupoles in Figs. (36-5), we can determine that the non-zero value of the induced quadrupoles is statistically significant. Although our numerical calculations suffer from Poisson fluctuations, the signal 

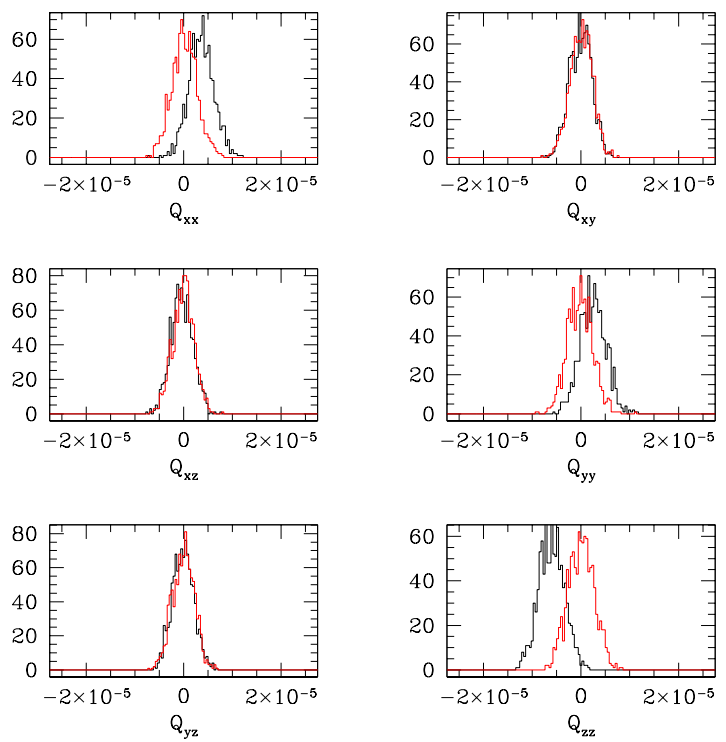

Fig. 4. Same as Fig. (3), but at $\nu=353 \mathrm{GHz}$.
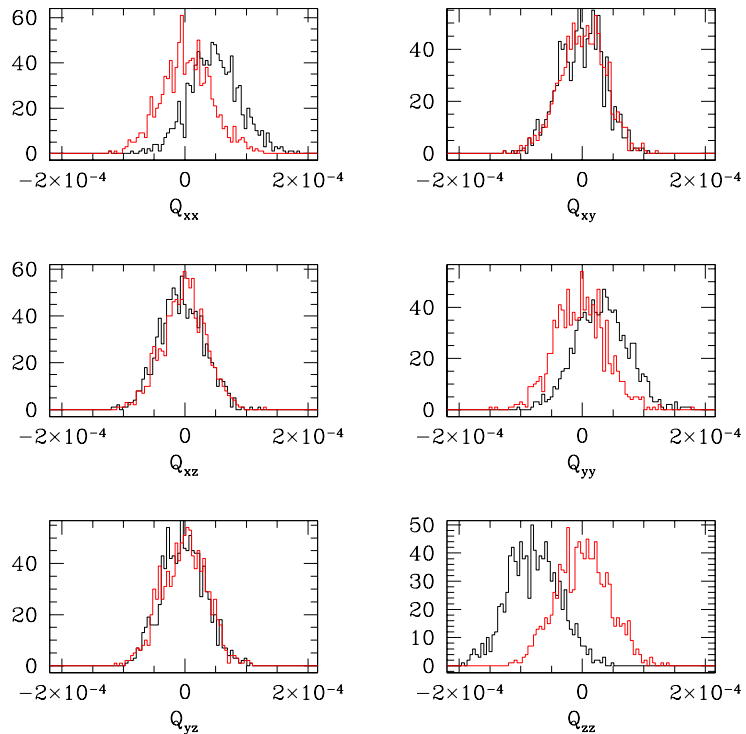

Fig. 5. Same as Fig. (3), but at $\nu=545 \mathrm{GHz}$.

from the real Oort Cloud will not exhibit the relatively large changes over an orbital period if our model for the size distribution of Oort Cloud objects and estimate of the total mass are reasonable. The quadrupole moments shown in Figs. (3 - 5) can be converted to a value of $C_{2}$, the Fourier space quadrupole power spectrum, using the formulae derived in Appendix A. The black points in Fig. 6 are the quadrupole power spectrum, $C_{\ell=2}$, as a function of frequency along with the expected Planck instrumental noise errorbar 10 . The expected

$\overline{10}$ The instrumental error bars appear anomalously low because we are accustomed 


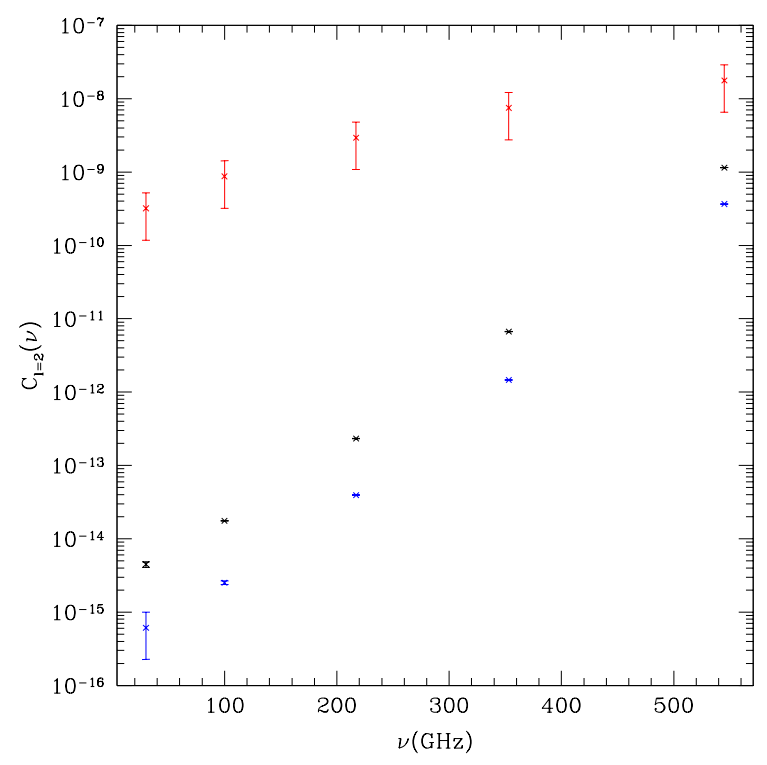

Fig. 6. The quadrupole power, $C_{\ell=2}$, at several different frequencies. The power spectrum is dimensionless since the Oort Cloud quadrupole moments are expressed in terms of the mean CMB intensity. The black points are the Oort Cloud power spectrum with error bars appropriate for Planck instrument noise. The blue points are the power spectrum produced by our model of the Scattered Disc. The red points are the theoretical power spectrum calculated with the best fit WMAP cosmology, corresponding to $C_{2}=1.76 \times 10^{-10}$ in terms of temperature fluctuations, with cosmic variance error bars. Since we chose to represent the fluctuations in terms of intensity and not temperature, the primordial temperature anisotropies do not produce a frequency independent power spectrum.

statistical fluctuations due to Poisson fluctuations in the number of observed Oort Cloud objects are much smaller for the models considered in this paper (see Table 2). Of course, the observability of the signal will primarily be affected by all of the astrophysical sources that emit non-blackbody radiation, such as the standard Galactic foregrounds, thermal Sunyaev-Zel'dovich emission, etc. The signal originating in our Solar System must be observed against this background. The full calculation of the power spectrum as function of frequency of these various backgrounds is beyond the scope of this paper.

Also shown in Fig. 6 is the quadrupole power spectrum of the Scattered Disc (blue points) and the theoretical power spectrum predicted by the WMAP cosmology model (red points). The theoretical power spectrum is frequency independent when expressed in terms of temperature fluctuations and has a value of $C_{2}=1.76 \times 10^{-10}$ (Hinshaw et al., 2006). Since we plot the intensity fluctuations, and not the temperature fluctuations, the theoretical power spectrum displays a slight frequency dependence.

to seeing the cosmic variance errors bar dominate at low $\ell$. 
Our assumption that the distributions of test particle mass and orbital elements are independent allows us to vertically scale the curves in Fig. 6] as we change $f_{A}$. The predictions for the power spectrum scale as $f_{A}^{2}$. Although we have separately plotted the quadrupole power spectra for the Oort Cloud and the Scattered Disc, in reality we will observe the combination of the two. We chose to separate the two contributions in Fig. 6 because the Scattered Disc is intrinsically aspherical and the Oort Cloud's asphericity is induced by a perturbation. Even if the Oort Cloud was perfectly spherical, the scattered disc, which is believed to smoothly compress from a spherical shape at the inner edge of the Oort Cloud into the Ecliptic Plane at the outer edge of the Kuiper Belt, will produce a minimum quadrupole moment.

While we calculate the corresponding quadrupole power spectrum values for comparision with CMB values, we should emphasize that the individual quadrupole moments of the induced spectral distortions contain phase information. It is assumed that the CMB temperature anisotropies constitute a Gaussian random field and the observed $a_{\ell m}$ 's are interpreted as being a random realization drawn from a Gaussian probability distribution function with variance $C_{\ell}$. In which case, the only meaningful information in a set of CMB data are the measured values of the $C_{\ell}$ 's. For the anisotropic spectral distortions induced by the Oort Cloud the particular values of the individual $a_{2 m}$ 's, or equivalently the $Q_{i j}$ (see Appendix $\mathrm{A}$ for relations that link these quantities), contain important information about the structure of the Oort Cloud. This is an important distinction to remember and one that we will explore in more detail in $\$ 5$.

The instrumental noise error bars appear anomalously small in Fig. 6. Typically on large scales cosmic variance dominates instrumental noise; for Planck the power spectrum should be cosmic variance limited until $\ell \approx 1500$. The error bars on the theoretical primordial quadrupole power spectrum in Fig. 6] are due to cosmic variance. Although the Oort Cloud and Scattered Disc power spectra are never distinguishable from the theoretical power spectrum at a single frequency, we can use the fact the primoridal CMB temperature anisotropies are frequency independent to utilize the low frequency data (where the Oort Cloud signal is effectively zero) to eliminate the cosmic variance uncertainity in the primordial temperature anisotropies at high frequency. Namely the low frequency temperature anisotropies can be used to exactly predict the high frequency temperature anisotropies 11 . The fact that cosmic variance limits our ability to measure the true power spectrum is irrelevant because we only care about the particular realization of the temperature anisotropies in our Universe. This should allow us to use the temperature anisotropies in a single frequency bin to remove them from the other frequencies and detect the Oort Cloud signal whenever it is larger than instrumental

\footnotetext{
${ }^{11}$ This statement depends on the level of foreground contamination in the low frequency bands
} 
noise.

\section{Bayesian Analysis}

The observed CMB can be used to constrain the parameters of the stellar encounter if a quadrupole, or any higher order moment, spectral distortion were to be detected. The posterior likelihood of the Oort Cloud and external perturbation parameters, collectively designated as $T$, given the observed data, designated as $D$, is

$$
\mathcal{L}(T \mid D) \propto \mathcal{L}(D \mid T) \mathcal{L}(T)
$$

where we have used Bayes' Theorem to invert the conditional probabilities. The external perturbation parameters depend on the mass of the perturber, $M_{p}$; the impact parameter of the encounter, $\mathbf{b}$; the velocity of the perturber with respect to the Solar System, $\mathbf{v}_{\text {rel }}$; and the absolute covering fraction of Oort Cloud objects, $f_{A}$. There exist degeneracies between some of these parameters. For example, the projection matrix, defined in Eq.(29), is invariant under the change $\hat{v} \rightarrow-\hat{v}$ since the force parallel to the trajectory cancels in the impulse approximation, and the total impulse only depends on the combination $M_{p} / b^{2} v_{r e l}$. Plots of the parameter constraints will directly display these degeneracies as the data is only sensitive to particular combinations, although the a priori probabilities will constrain these parameters to some extent.

The likelihood function of the data given the stellar encounter parameter can be expressed as a functional integration over the true CMB signal (S), which will be a combination of primordial temperature anisotropies and Oort Cloud emission,

$$
\mathcal{L}(D \mid T)=\int d S \mathcal{L}(D \mid S) \mathcal{L}(S \mid T)
$$

where the likelihood of the data given the true CMB signal is simply set by instrument noise (Knox, 1995; Dodelson, 2003)

$$
\mathcal{L}(D \mid S)=\frac{1}{\sqrt{2 \pi \operatorname{det} N}} e^{-(d-a)^{\dagger} \mathbf{N}^{-1}(d-a) / 2}
$$

where $\mathbf{N}$ is the noise covariance matrix, $d$ is the observed signal and $a$ is the true underlying CMB signal. The observed signal is not uniquely determined by the underlying theory which only specifies the probability distribution func-

tion of which the signal is a random realization. The likelihood function of the CMB temperature anisotropies is

$$
\mathcal{L}(S \mid T)=\frac{1}{\sqrt{2 \pi \operatorname{det} C}} e^{-\boldsymbol{a}^{\dagger} \mathbf{C}^{-1} \boldsymbol{a} / 2}
$$


where $\mathbf{C}$ is the signal covariance matrix. The signal covariance matrix is defined as the expectation value of $\mathbf{C}=\left\langle\boldsymbol{a} \boldsymbol{a}^{\dagger}\right\rangle$, with a similar definition for the noise covariance matrix $\mathbf{N}$. The likelihood of the Oort Cloud signal given the theory is set a probability distribution is determined by Poisson fluctuations as discussed above in $\$ 2.1$.

The a priori probability of Oort Cloud and stellar perturbation parameters should not be modeled as the product of the individual parameter distributions. Observations of the local Solar neighborhood have found that different stellar spectral types have different number densities and different velocity ellipsoids (Binney \& Merrifield, 1998; King et al., 1990). We should be mindful that the Sun's local environment might have been quite different in the past as the Sun moves through different Galactic environments. The a priori distribution can be written in terms of conditional probabilities as

$$
\mathcal{L}(D)=p\left(b \mid v_{r e l}, M\right) p(\hat{b} \mid \hat{v}) p\left(\mathbf{v}_{\text {rel }} \mid M\right) p(M) p\left(f_{A}\right)
$$

where $\mathrm{p}(\mathrm{M})$ is the distribution of stellar masses, averaged according to stellar evolutionary theory as

$$
p(M)=\Psi(M) \frac{t(M)}{\Delta t},
$$

where $\Psi(M)$ is the appropriate initial mass function, $t(M)$ is the lifetime of stars of mass $M$ and $\Delta t$ is the lifetime of the Solar System. The distribution of velocities is assumed to follow a Schwartzschild distribution (Binney \& Tremaine, 1987)

$$
p\left(\mathbf{v}_{\text {rel }} \mid M\right)=\frac{1}{(2 \pi)^{3 / 2} \operatorname{det} \boldsymbol{\Sigma}} e^{-\mathbf{v} \boldsymbol{\Sigma} \mathbf{v} / 2}
$$

with the velocity ellipsoid $(\boldsymbol{\Sigma})$ depending on the stellar spectral type (King et al., 1990; Binney \& Merrifield, 1998). As discussed in \$3, the direction of the impact parameter is the determined by the direction of the encounter velocity up to a phase angle. The magnitude of the impact parameter depends on the number density of stars of a given mass and their relative velocity with respect to the Sun 12 . Given these parameters, the distribution of impact parameters can be calculated as outlined in \$2.4. The a priori probability of $f_{A}$ is completely unknown, except that an upper limit can be set using constraint on the mean CMB spectrum distortion (Babich et al., 2007). We can consider the CMB power spectrum, needed to specify the likelihood function of the extragalactic temperature anisotropies, as known.

If a quadrupole spectral distortion in the CMB is definitively measured then this formalism can be exploited to constrain the closest stellar encounter. There are similiar hopes to constrain such a stellar perturbation by using the

\footnotetext{
$\overline{12}$ Gravitational focusing will induce an additional correlation between the distribution of $b$ and $v_{r e l}$, but we ignore it here since gravitational focusing is not consistent with our use of the impulse approximation.
} 
extreme orbits of Sedna and analogs that should be discovered in the future. It remains to be seen if this goal can actually be accomplished as the associated CMB spectral distortion moments suffer from large statistical fluctuations and the initial conditions of the Sedna-like objects are unknown.

\section{Conclusions}

We analyzed the effect of a close stellar encounter on the shape of the Oort Cloud and the subsequent anisotropic CMB spectral distortions that would result. Much about the Oort Cloud is unknown and our calculations in this paper are subject to these uncertainities. We have strived to adopt conservative estimates for our mass model of the Oort Cloud and have highlighted the resulting uncertainities. The most likely minimum impact parameter over the past 1 Gyr for the present-day local Solar neighborhood is $b=2100 \mathrm{AU}$. Assuming the perturber is a Solar mass star traveling with relative velocity $30 \mathrm{~km} \mathrm{~s}^{-1}$ with respect to the Sun, the perturbation will produce frequency-dependent quadrupole moments in the observed CMB of $C_{2}=4.5(3.5) \times 10^{-15}, 6.7(1.1) \times 10^{-12}, 1.1(0.11) \times 10^{-9}$ at $\nu=30,353,545 \mathrm{GHz}$, respectively in the intensity and (temperature) fluctuations. The asphericity in the Oort Cloud that leads to our signal will produce spectral distortions in the $\mathrm{CMB}$ on all angular scales. Our calculation can be extended to the octopole $(\ell=3)$, or any higher moment, without much difficulty. Moreover the signal will be highly non-Gaussian and will induce correlations between the power spectra on different scales. These features may help in the detection of the signal. Using data from scales smaller than quadrupole will also help in disentangling the signal from the Galactic foreground emission.

The Planck Satellite 13 will have nine bands of frequency coverage between $\nu=30 \mathrm{GHz}$ and $857 \mathrm{GHz}$, with the $\nu=353 \mathrm{GHz}$ band the highest with reasonable estimated detector noise. Additionally, the recently launched farinfrared satellite Akari, originally Astro-F, has frequency coverage down to $2 \mathrm{THz}$ (Pearson et al., 2001) and might be able to search for the signal from the closest and hottest Oort Cloud objects.

Finally, we described a Bayesian method to use observed anisotropic spectral distortions to constrain the trajectory of the closest stellar perturber. The Bayesian method requires that the direction of the quadrupole, or equivalently the phase information in the quadrupole moments, does not drastically change over timescales comparable to the period of the Oort Cloud objects. The associated fluctuations are due to Poisson fluctuations in the number of Oort Cloud objects at any position in the outer Solar System. We quantified

\footnotetext{
$\overline{13}$ www.rssd.esa.int/Planck/
} 
these fluctuations and determined that they are important when interpreting our calculations due to the relatively small number of test particles $\left(10^{9}\right)$ we used. However, we estimated that the real Oort Cloud has approximately $10^{28}$ objects and the interpretation of the real quadrupole moments would not be affected by these statistical fluctuations. Therefore the direction of the quadrupole of CMB spectral distortions, if ever observed, could be used to constrain the properties of the closest stellar encounter with the Solar System.

\section{Acknowledgements}

We would like to thank M. Brown, P. Goldreich, M. Holman, Z. Lienhardt and P. Weissman for helpful conversations and L. Dones for sharing an unpublished manuscript. DB thanks the hospitality of the Harvard Institute for Theory and Computation where some of this work was completed and acknowledges financial support from the Betty and Gordon Moore Foundation.

\section{A Angular Moment Definitions}

In this appendix we review the relationships between the various descriptions of the angular moments of a distribution on a sphere, in particular the quadrupole moments which are discussed in this paper. It is advantageous to describe the CMB temperature anisotropies in a basis of spherical harmonics because of their orthonormality properties on the sphere. A function defined over the sphere, $A(\hat{\boldsymbol{n}})$, can be expanded in a spherical harmonic basis as

$$
a_{\ell m} \equiv \int d^{2} \hat{\boldsymbol{n}} Y_{\ell m}^{*}(\hat{\boldsymbol{n}}) A(\hat{\boldsymbol{n}})
$$

Since the CMB temperature anisotropies are assumed to be described by a Gaussian random field the particular values of the $a_{\ell m}$ are not meaningful, only the statistical properties of the expansion coefficients carry any information about the underlying cosmology. Therefore, it is customary to calculate the ensemble average of the $a_{\ell m}$ covariance matrix

$$
\left\langle a_{\ell_{1} m_{1}} a_{\ell_{2} m_{2}}\right\rangle \equiv \delta_{\ell_{1}, \ell_{2}} \delta_{m_{1},-m_{2}}(-1)^{m_{1}} C_{\ell_{1}}
$$

which can be related to an isotropic 14 quantity, the power spectrum, $C_{\ell}$. This identity can be used to motivate an observational definition of $C_{\ell}$

$$
C_{\ell}=\frac{1}{2 \ell+1} \sum_{m=-\ell}^{\ell}(-1)^{m} a_{\ell m} a_{\ell-m} .
$$

\footnotetext{
${ }^{14}$ Invariant under rotations of our coordinate system. For example, it is invariant with respect to different choices of our reference direction.
} 
We will now relate these quantities to the Cartesian multipole moment expansions utilized in our paper and defined in Eq. (14). Expressing the Cartesian directional vectors in spherical coordinates,

$$
\begin{aligned}
\hat{n}_{x}+i \hat{n}_{y} & =\sin \theta e^{i \phi}, \\
\hat{n}_{z} & =\cos \theta .
\end{aligned}
$$

Then the five $\ell=2$ spherical harmonics can be expressed as

$$
\begin{aligned}
Y_{22}(\hat{\boldsymbol{n}}) & =\sqrt{\frac{3}{8}} \sqrt{\frac{5}{4 \pi}}\left(\hat{n}_{x}+i \hat{n}_{y}\right)^{2}, \\
Y_{21}(\hat{\boldsymbol{n}}) & =-\sqrt{\frac{3}{2} \sqrt{\frac{5}{4 \pi}}} \hat{n}_{z}\left(\hat{n}_{x}+i \hat{n}_{y}\right), \\
Y_{20}(\hat{\boldsymbol{n}}) & =\frac{3}{2} \sqrt{\frac{5}{4 \pi}}\left(\hat{n}_{z}^{2}-\frac{1}{3}\right), \\
Y_{2-1}(\hat{\boldsymbol{n}}) & =\sqrt{\frac{3}{2}} \sqrt{\frac{5}{4 \pi}} \hat{n}_{z}\left(\hat{n}_{x}-i \hat{n}_{y}\right), \\
Y_{2-2}(\hat{\boldsymbol{n}}) & =\sqrt{\frac{3}{8}} \sqrt{\frac{5}{4 \pi}}\left(\hat{n}_{x}-i \hat{n}_{y}\right)^{2} .
\end{aligned}
$$

From these definitions relationships between the $a_{2 m}$ and $Q_{i j}$ can be derived.

$$
\begin{aligned}
a_{22} & =\sqrt{\frac{3}{8}} \sqrt{\frac{5}{4 \pi}}\left(Q_{x x}-Q_{y y}-2 i Q_{x y}\right), \\
a_{21} & =-\sqrt{\frac{3}{2}} \sqrt{\frac{5}{4 \pi}}\left(Q_{x z}-i Q_{y z}\right), \\
a_{20} & =\frac{3}{2} \sqrt{\frac{5}{4 \pi}} Q_{z z} \\
a_{2-1} & =\sqrt{\frac{3}{2}} \sqrt{\frac{5}{4 \pi}}\left(Q_{x z}+i Q_{y z}\right), \\
a_{2-2} & =\sqrt{\frac{3}{8}} \sqrt{\frac{5}{4 \pi}}\left(Q_{x x}-Q_{y y}+2 i Q_{x y}\right) .
\end{aligned}
$$

The power spectrum can be related to the Cartesian quadrupole moments through Eq. A.3

$$
C_{2}=\frac{3}{4 \pi}\left(Q_{x x}^{2}+Q_{z z}^{2}+Q_{x y}^{2}+Q_{x z}^{2}+Q_{y z}^{2}+Q_{x x} Q_{z z}\right)
$$

The expectation value of this expression should contain both the mean and variance of the $Q_{i j}$ as terms. However, consistent with our calculation that Poisson fluctuations are not important we will evaluate Eq. (A.16) with only the means of the $Q_{i j}$. 


\begin{tabular}{ccc}
\hline \hline$\nu$ & $\sigma / T_{\mathrm{CMB}} \times 10^{-6}$ & $F W H M(\operatorname{arcmin})$ \\
\hline $30 \mathrm{GHz}$ & 2.0 & 33 \\
$100 \mathrm{GHz}$ & 2.5 & 9.5 \\
$217 \mathrm{GHz}$ & 4.8 & 5.0 \\
$353 \mathrm{GHz}$ & 14.7 & 5.0 \\
$545 \mathrm{GHz}$ & 147 & 5.0 \\
\hline \hline
\end{tabular}

Table B.1

Instrument noise parameters adopted for this paper. These values were taken from the Planck Science webpage (www.rssd.esa.int/Planck/).

\section{B Instrument Noise}

Finally, we describe the instrument noise values that were adopted in this paper. In temperature units the instrument noise for the observation of $C_{\ell}$ is (Knox, 1995)

$$
C_{\ell}^{N}=\sqrt{\frac{2}{2 \ell+1}} \frac{4 \pi \sigma^{2}}{N_{p i x}} e^{\ell^{2} \sigma_{B}^{2}},
$$

where the beam width is related to the FWHM as $\sqrt{8 \ln 2} \sigma_{B}=\mathrm{FWHM}, N_{p i x}$ is the number of observed pixels and $\sigma$ is the noise per pixel in temperature units. The temperature fluctuation can be related to an intensity fluctuations as follows,

$$
\Delta I_{\nu}=\left.\frac{\partial B_{\nu}(T)}{\partial T}\right|_{T_{\mathrm{CMB}}} \Delta T_{\nu}
$$

Table $\mathrm{B}$ lists the noise parameters used in this paper.

\section{References}

Adams F. C., \& Laughlin G. 2001, Icarus, 150, 151

Aguilar L. A., \& White S. D. M. 1985, ApJ, 295, 374

Babich D., \& Loeb A. 2007, MNRAS, 374, L24

Babich D., Blake C. \& Steinhardt C. 2007, submitted to ApJ

Backman, D. E., \& Paresce, F. 1993, Protostars and Planets III, 1253

Bernstein G.M. et al. 2004, AJ, 128, 1364

Binney J., \& Merrifield M. 1998, Galactic astronomy Princeton, NJ : Princeton University Press, pp. 603-689

Binney J. \& Tremaine S. 1987, Princeton, NJ, Princeton University Press, pp. 433-440, pp. 211-214

Brown M. E. 2007, personal communication

Brown M. E., Trujillo C. A., \& Rabinowitz D. L. 2004, ApJ, 617645

Burns, J. A., Lamy, P. L., \& Soter, S. 1979, Icarus, 40, 1 
Charnoz S. \& Morbidelli A. 2003, Icarus, 166, 141

Charnoz S. \& Morbidelli A. 2006, AAS/Division for Planetary Sciences Meeting Abstracts, 38, \#34.04

Danby J. 1962, New York: Macmillan, 1962,

Delsemme A. H. 1987, A\&A, 187, 913

Dodelson S. 2003, Modern cosmology Amsterdam (Netherlands): Academic Press, p. 216

Dones L. et al. 2004, ASPC 323, 371

Dones L. et al. 2007, submitted to Icarus

Duncan M., Quinn T., \& Tremaine S. 1987, AJ, 94, 1330

Fixsen D. J., Hinshaw G., Bennett C. L., \& Mather, J. C. 1997, ApJ, 486, 623

García-Sánchez J., et al. 1999, AJ, 117, 1042

García-Sánchez J., et al. 2001, A\&A, 379, 634

Goldreich P., Lithwick Y., \& Sari R. 2004, ARA\&A, 42, 549

Greenberg J. M. 1978, Cosmic Dust, 187

Heisler J. \& Tremaine S. 1986, Icarus, 65, 13

Hester J. J., Desch S. J., Healy K. R., \& Leshin L. A. 2004, Science, 304, 1116

Hinshaw G., et al., arXiv:astro-ph/0603451

Hut P., \& Tremaine S. 1985, AJ, 90, 1548

Ida S., Larwood J., \& Burkert A. 2000, ApJ, 528, 351

Jewitt, D., \& Luu, J. 1998, AJ, 115, 1667

Kenyon S. J., \& Bromley B. C. 2002, AJ, 123, 1757

Kenyon S. J., \& Bromley B. C. 2004, Nature, 432, 598

King I., Gilmore G., \& van der Kruit P. C. 1990, The Milky Way As Galaxy, University Science Books, pp. 227-247

Knox L. 1995, PRD, 52, 4307

Lada C. J., \& Lada E. A. 2003, ARA\&A, 41, 57

Landau, L. D., \& Lifshitz, E. M. 1999, Course of Theoretical Physics - Vol.5, Statistical Physics, Part 1 Butterworth-Heinemann: Oxford

Larwood J. D. 1997, MNRAS, 290, 490

Looney L. W., Tobin J. J., \& Fields B. D. 2006, ApJ, 652, 1755

Marsden B. G. \& Sekanina Z. 1971 AJ, 76, 1135

Mather, J. C., et al. 1999, ApJ, 512, 511

Mumma M. J., Weissman P. R. \& Stern S. A. 1993, Protostars and Planets III, 1177

Murray C. D., \& Dermott S. F. 2000, Solar System Dynamics, Cambridge, UK: Cambridge University Press, 2000, p.54

Ostriker, E. C. 1994, ApJ, 424, 292

Oort J. H. 1950, Bull. Astro. Inst. Netherlands, 11, 91

Pan M. \& Sari R. 2005, Icarus, 173, 342

Pearson C. P., et al. 2001, MNRAS, 324, 999

Rafikov R. R. 2004, AJ, 128, 1348

Stern A. \& Weissman P. 2001, Nature 409, 6820, 589

Stern A. 2003, Nature 424, 6949, 639

Tremaine S. 2005, ApJ, 625, 143 
Weidenschilling S. J. 1977, MNRAS, 180, 57

Weissman P. R. 1992, BAAS, 24, 1063

Weissman P. R. 1990, Nature, 344, 825

Yabushita S., Hasegawa I., \& Kobayashi K. 1982, MNRAS, 200, 661 